\title{
MEASUREMENT OF THE PION POLARIZABILITIES AT COMPASS
}

\author{
A. V. GUSKOV ON BEHALF ON THE COMPASS COLLABORATION \\ Dzhelepov Laboratory of Nuclear Problems, Joint Institute for Nuclear Research, Dubna, Russia \\ *E-mail: avg@nusun.jinr.ru
}

\begin{abstract}
The electromagnetic structure of pions is probed in $\pi \gamma$ Compton scattering in inverse kinematics (Primakoff effect) and described by the electric $\left(\alpha_{\pi}\right)$ and magnetic $\left(\beta_{\pi}\right)$ polarizabilities, that depend on the rigidity of pion's internal structure as a composite particle. Values for pion polarizabilities can be extracted from the comparison of the theoretically predicted (under approximation of unstructured pion) cross section of Primakoff scattering and the measured cross section. The high beam intensity, good spectrometer resolution, the high rate capability, the high acceptance and possibility to use pion and muon beams, that are unique to the COMPASS experiment provide the tools to measure precisely the pion polarizabilities in the $\pi^{-}+(A, Z) \rightarrow \pi^{-}+(A, Z)+\gamma$ Primakoff reaction. This cross section is related to the cross section of Compton scattering on pion. A precise tracking system, electromagnetic and hadron calorimeters provide good conditions for Primakoff events selection and background suppression. The pion polarizability measurement was performed with a $\pi^{-}$beam of $190 \mathrm{GeV}$. Most of the statistics was collected with a $3 \mathrm{~mm}$ thick lead target, along with smaller samples using carbon, copper, $1.6 \mathrm{~mm}$ lead and empty target. Total statistics collected with lead target is about 60000 events.
\end{abstract}

Keywords: pion polarizabilities; Primakoff scattering; chiral theory.

\section{Pion polarizabilities: theory and experiment}

Pion polarizabilities characterize pion interacting as a complex $q \bar{q}$ system with external electromagnetic fields. They are fundamental parameters of pion physics and the comparison of theoretically predicted and directly measured values provides stringent tests for various theoretical models. Chiral perturbation theory $(\chi P T)$ is one of the most successful tools in describing low energy hadron properties and provides for the two-loop approximation the values ${ }^{1}$ :

$$
\begin{gathered}
\alpha_{\pi}=2.4 \pm 0.5 \times 10^{-4} \mathrm{fm}^{3} \\
\beta_{\pi}=-2.1 \pm 0.5 \times 10^{-4} \mathrm{fm}^{3}
\end{gathered}
$$

The predictions of other theoretical models like dispersion sum rules, QCD sum rule, lattice calculations lie in the range from 2.4 to 8.0 for $\alpha_{\pi}$ and from -8.0 to -2.1 for $\beta_{\pi}$ in units of $10^{-4} \mathrm{fm}^{3}$. For the experimental measurement of charged pion polarizabilities three physical processes are used:
- Primakoff reaction:

$$
\pi+(A, Z) \rightarrow \pi+(A, Z)+\gamma
$$

- Pion photoproduction:

$$
\gamma+(A, Z) \rightarrow \gamma+(A, Z)+\pi
$$

- $\pi^{+} \pi^{-}$pair production in $e^{+} e^{-}$interactions:

$$
\gamma+\gamma \rightarrow \pi^{+} \pi^{-}
$$

\begin{tabular}{|c|c|c|}
\hline Data & value & $10^{-4} \mathrm{fm}^{3}$ \\
\hline \multicolumn{3}{|c|}{$\pi+(A, Z) \rightarrow \pi+(A, Z)+\gamma$} \\
\hline Protvino $^{3}$ & $\begin{array}{c}\alpha_{\pi} \\
(A, Z) \rightarrow\end{array}$ & $\begin{array}{l}6.8 \pm 1.4 \pm 1.2 \\
+(A, Z)+\pi\end{array}$ \\
\hline Lebedev ${ }^{4}$ & $\alpha_{\pi}$ & $20 \pm 12$ \\
\hline Mami A2 ${ }^{5}$ & $\begin{array}{c}\alpha_{\pi}-\beta_{\pi} \\
\gamma+\gamma\end{array}$ & $\begin{array}{l}11.6 \pm 1.5 \pm 3.0 \pm 0.5 \\
\pi^{+} \pi^{-}\end{array}$ \\
\hline PLUTO $^{6}$ & $\alpha_{\pi}$ & $19.1 \pm 4.8 \pm 5.7$ \\
\hline DM1 7 & $\alpha_{\pi}$ & $17.2 \pm 4.6$ \\
\hline DM2 ${ }^{8}$ & $\alpha_{\pi}$ & $26.3 \pm 7.4$ \\
\hline Mark II ${ }^{9}$ & $\alpha_{\pi}$ & $2.2 \pm 1.6$ \\
\hline
\end{tabular}

Table 1. Experimental results for charged pion polarizabilities 
The experimental results are presented in Table 1. Significant disagreement between measured values and large statistical and systematical uncertainties don't allow to confirm or disprove the $\chi P T$ prediction. New more precise measurements are needed. Precise measurement of pion polarizabilities with the Primakoff reaction is one of the goals of COMPASS experiment ${ }^{2}$.

\section{Primakoff reaction.}

The Primakoff reaction is a process in which charged pion electromagnetically scatters on a nuclear target under emission of a hard photon. It can be treated as Compton scattering of virtual photon provided by the nucleus on the pion. The cross section of this reaction in the rest frame of the incoming pion can be represented as:

$$
\begin{gathered}
\frac{d^{3} \sigma}{d t d \omega d \cos \theta}=\frac{2 \alpha^{3} Z^{2}}{m_{\pi}^{2} \omega} \frac{Q^{2}-Q_{0}^{2}}{Q^{4}} F_{A}\left(Q^{2}\right)^{2} \times(4) \\
\times\left(F_{\pi \gamma}^{p l}+\frac{m_{\pi} \omega^{2}}{\alpha} \frac{\alpha_{\pi}\left(1+\cos ^{2} \theta\right)+2 \beta_{\pi} \cos \theta}{\left(1+\frac{\omega}{m_{\pi}}(1-\cos \theta)\right)^{3}}\right)
\end{gathered}
$$

where $m_{\pi}$ is the pion mass, $\omega$ is the energy of the virtual photon, $\theta$ is the photon scattering angle, $Q$ is the momentum transferred to nucleus, $Q_{0}=\frac{m_{\pi} \omega}{P_{\text {beam }}}, F_{A}\left(Q^{2}\right)$ is the electromagnetic form factor of nucleus. $\left(F_{A}\left(Q^{2}\right) \approx 1\right.$ for small $\left.Q^{2}\right), F_{\pi \gamma}^{p l}$ represents the Compton term for a point-like spin-less particle. The dependency of the Primakoff cross section on pion polarizabilities allows to use the reaction for separate measurement of $\alpha_{\pi}$ and $\beta_{\pi}$. The small momentum transfer to the nucleus $\left.\left(Q^{2}<<m_{\pi}^{2}\right)\right)$ can be used as the signature of the process.

\section{Primakoff reaction studies at COMPASS}

Primakoff reaction studies at COMPASS during the pilot hadron run in 2004 were performed with a $190 \mathrm{GeV} \pi^{-}$beam and a $3 \mathrm{~mm}$ lead target. Additional samples with copper, carbon and empty targets and a $190 \mathrm{GeV} \mu^{-}$beam can be used to study background processes and estimate systematic errors. COMPASS provides unique conditions for the investigation of the Primakoff process: silicon detectors (spacial resolution better than $16 \mu \mathrm{m}$ ) for the vertex position reconstruction and for the measurement of the pion scattering angle, an electromagnetic calorimeter for the photon 4-momentum reconstruction and a magnetic spectrometer for the determination of the scattered pion's momentum.

\subsection{Primakoff trigger}

Two types of triggers were introduced to enrich Primakoff events: One selects events with at least one scattered particle with a momentum below $150 \mathrm{GeV} / c$ in coincidence with a high energy photon. The scattered pion with a momentum $20 \div 140 \mathrm{GeV} / c$ is detected by a trigger hodoscope. In addition, an energy deposition larger than $\sim 50 \mathrm{GeV}$ in the electromagnetic calorimeter and an energy deposition smaller than $\sim 18 \mathrm{GeV}$ in the hadron calorimeter are required. The other trigger was introduced to enhance the region of pion momenta below $90 \mathrm{GeV} / c$. Only an energy deposition above $100 \mathrm{GeV}$ in the electromagnetic calorimeter is required for it.

\subsection{Event selection}

The candidate Primakoff events are selected with the following requirements:

- existence of the beam track;

- only one secondary track with momentum and negative charge, reconstructed in the silicon detectors;

- only one cluster in the electromagnetic calorimeter with the an energy above 7 $\mathrm{GeV}$;

- vertex position close to the nominal target position (Fig.1); 
- pion scattering angle $>0.2 \mathrm{mrad}$ (to exclude effects related to multiple scattering of the beam pion in the lead target).

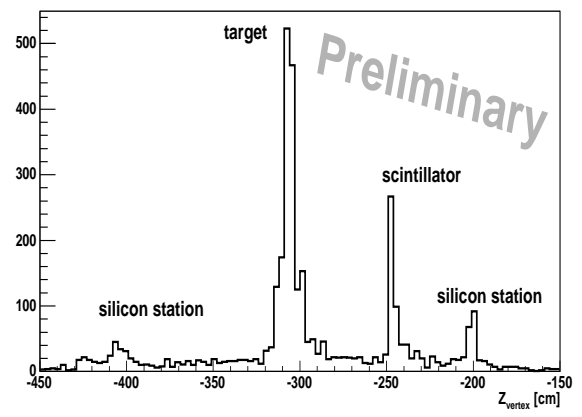

Fig. 1. Vertex position reconstruction.

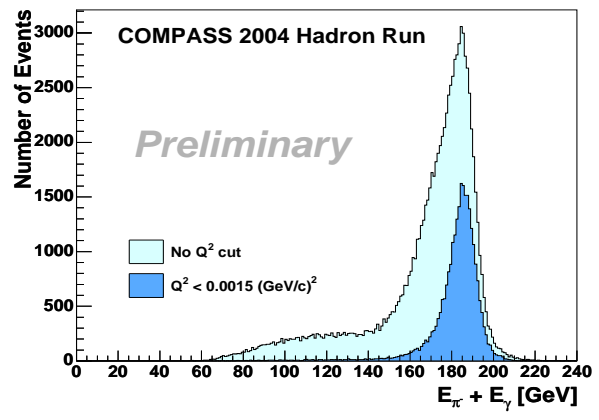

Fig. 2. Exclusivity peak.

\subsection{Energy balance}

The energy transferred to the nucleus is much less than a setup resolution. Under zero transfered energy approximation for Primakoff scattering $E_{\text {beam }}=E_{\text {ycluster }}+$ $E_{\text {mscatt. }}$, see Fig.2. The width of the reconstructed beam energy peak is about 7 $\mathrm{GeV}$ and the main contribution to the peak width is due to the electromagnetic calorimeter energy resolution. The exclusivity of Primakoff events is enforced by accepting only events for which the reconstructed beam en$\operatorname{ergy}\left(E_{\gamma \text { cluster }}+E_{\pi \text { scatt. }}\right)$ is in the range 165 $215 \mathrm{GeV}$.

\section{4. $Q^{2}$-distribution}

For the calculation of the scattered photon 4momentum we can neglect the energy transferred to the lead nucleus (but not the momentum). In this way the photon energy $E_{\gamma}=E_{\text {beam }}-E_{\pi s c a t t}$ and $Q^{2}=\left(E_{\pi s c a t t} \times\right.$

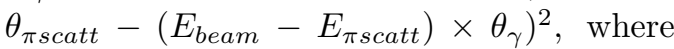
$\theta_{\pi s c a t t}$ and $\theta_{\gamma}$ are the scattering angles for the pion and the photon. A small beam momentum spread and the precise measurement of scattered pion momentum allow to obtain a smaller uncertainty for the photon energy than using the energy information from the calorimeter. The obtained $Q^{2}$ distribution is presented in Fig.3.

In addition to the electromagnetic scattering process with the $\pi^{-}+\gamma$ final state we have diffractive scattering processes with the same signature, for which the transfered momentum is not zero. The diffractive scattering process produces some background level in the region of large $Q^{2}$ while the peak at $Q^{2}=0$ corresponds to Primakoff events. The width of this peak is fully defined by the setup resolution for $Q^{2}$. For Primakoff and diffractive scattering separation we use the cut $Q^{2}<1.5 \times 10^{-3}(\mathrm{GeV} / \mathrm{c})^{2}$. This $Q^{2}$ approximately corresponds to equal contributions from Primakoff and diffractive scattering. The diffractive background for higher 4momentum transfers and the clear Primakoff signal for $Q^{2}<1.5 \times 10^{-3}(\mathrm{GeV} / \mathrm{c})^{2}$ can be seen in Fig.3.

\subsection{Final state invariant mass}

The invariant mass spectrum (Fig.4) of the reconstructed $\pi^{-} \gamma$ system after all described selection cuts shows a peak at $700 \mathrm{MeV}$ corresponding to $\rho^{-}$decaying into $\pi^{-} \pi^{0}$. In case of the loss of one photon from the decay $\pi^{0} \rightarrow \gamma+\gamma$ or in case of a small angle 


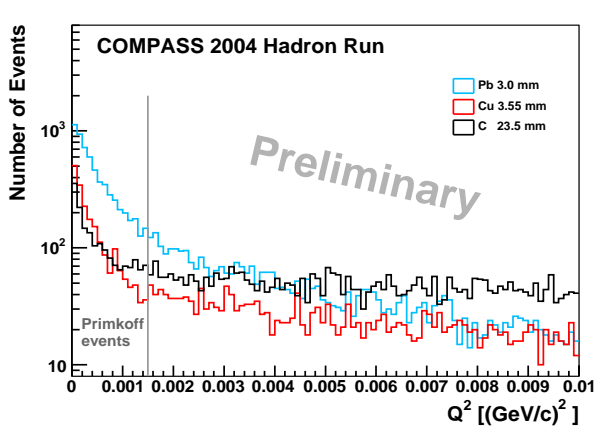

Fig. 3. $Q^{2}-$ distribution

between the photons and only one cluster reconstructed in the electromagnetic calorimeter the $\rho^{-}$-decay events have the same signature as a Primakoff events. Taking into account that the nominal $\rho$-meson width is $150 \mathrm{MeV}$, events with $M_{\pi \gamma}>4 \times m_{\pi^{-}}$possibly will be excluded from the polarizabilities analysis.

A similar background also can be observed from the decay of the beam kaons (4\%) $K^{-} \rightarrow \pi^{-} \pi^{0}$. Its contribution can be studied with empty target samples.

\subsection{Prospects for pion polarizability measurement at COMPASS}

Taking into account the current efficiency of the Primakoff event selection we estimate a statistics of Primakoff events for $\pi^{-}$beam and lead target of 60000 events. About 30000 of these events have a relative energy of the scattered photon in the range $0.5<\frac{E_{\gamma}}{E_{\text {beam }}}<0.9$ (for comparison, the statistics of the Protvino experiment in this range was 7000 events) (Fig.5). COMPASS also covers the range $0.25<\frac{E_{\gamma}}{E_{\text {beam }}}<0.5 \mathrm{im}$ portant for independent determination of $\alpha_{\pi}$ and $\beta_{\pi}$ but not covered by the Protvino experiment. Big statistics, samples with muon beam and different targets provide a chance to decrease statistical and systematic errors of pion polarizability measurement.

\section{References}

1. U. Bürgi, Phys. Lett. B377, 147 (1996)

2. COMPASS collaboration, Proposal for a Common Muon Proton Apparatus for Structure and Spectroscopy, http://wwwcompass.cern.ch

3. Yu. M. Antipov et al., Phys. Lett. B121, 445 (1983)

4. T. A. Aybergenov et al.,Czech. J. Phys. 36, 948 (1986)

5. J. Ahrens et al.,Eur. Phys. J. A 23, 113 (2005)

6. C. Berger et al., (PLUTO collaboration) $Z$. Phys. C 26, 199 (1984)

7. A. Courau et al., (DM1 collaboration) Nucl. Phys. B271, 1 (1986)

8. Z. Ajaltoni et al.,(DM2 collaboration) Phys. Lett. B377, 147 (1996)

9. J. Boyer et al. (Mark II collaboration) Phys. Rev. D 42, 1350 (1990)

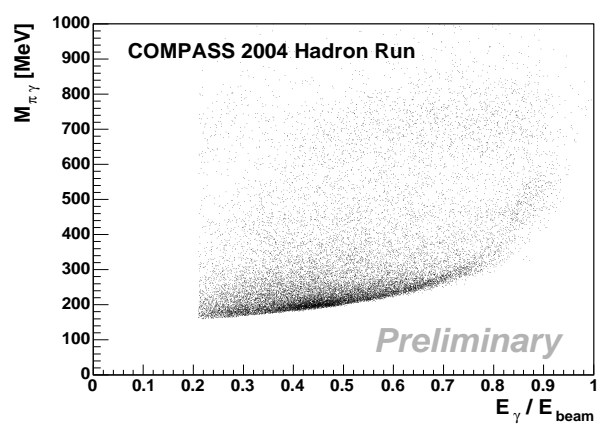

Fig. 5. $\quad \pi \gamma$ invariant mass

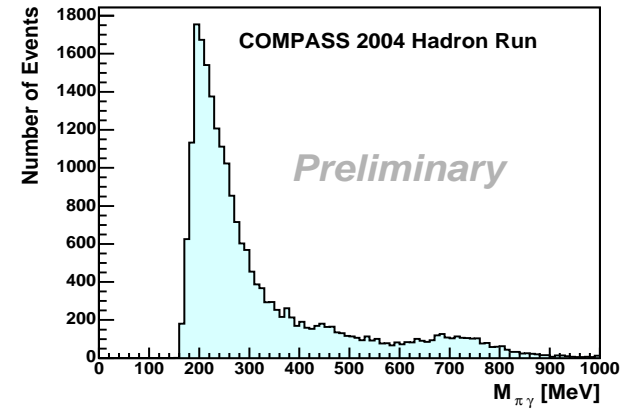

Fig. 4. $\quad \pi \gamma$ invariant mass 